\title{
Transverse Feedback System With Digital Filter
}

\author{
V. M. Zhabitsky \\ Joint Institute for Nuclear Research, 141980 Dubna, Moscow Region, Russia \\ I. L. Korenev and L. A. Yudin \\ Moscow Radiotechnical Institute, 113519 Moscow, Russia
}

\begin{abstract}
Main theoretical results for a transverse feedback system with a digital IIR-filter (infinite duration impulse response filter) and FIR-filter (finite duration impulse response filter) are described. The $Z$-transform method is used to solve the problem of the beam dynamics in the accelerator with a digital feedback. The analytical solution for the damping time and for the eigen frequencies are obtained and the system stability analyzed.
\end{abstract}

\section{INTRODUCTION}

The transverse feedback systems (TFS) are used in synchrotrons to damp the coherent transverse beam oscillations. In these systems the kicker (DK) corrects the beam angular according to the beam deviation from the closed orbit in the pick-up (PU) location at each turn. A classical TFS consists of one PU and one DK per plane. These systems have been used widely and provide an amplitude decrease of $25 \%$ per revolution [1]. In order to suppress fast resistive wall instability in UNK-1 (Serpukhov, Russia) [2] a more effective system is studied and developed [3]. It consists of two PU and two DK per plane connected by a feedback circuit with a digital filter and delay. The digital filters are used in a classical TFS [4] and designed for a fast TFS [5] to remove the revolution frequency harmonics. Feedbacks with digital filters have essential advantages for new large accelerators such as UNK, LHC, SSC where the revolution period is $70 \div 290 \mu s$ and the digital procedures for signal transformations can be realizcd with signal processors. This article is based on studies of TFS for UNK-1 $[3,5]$ and LHC $[6]$.

\section{Basic EqUATIONS}

A feedback system consists of PU and DK connected by circuit with a preamplifier, a filter, a power amplifier and a delay $\tau$ (Fig.la). If bunch coupling, which happens due to resistive wall instability, is neglected, the matrix method
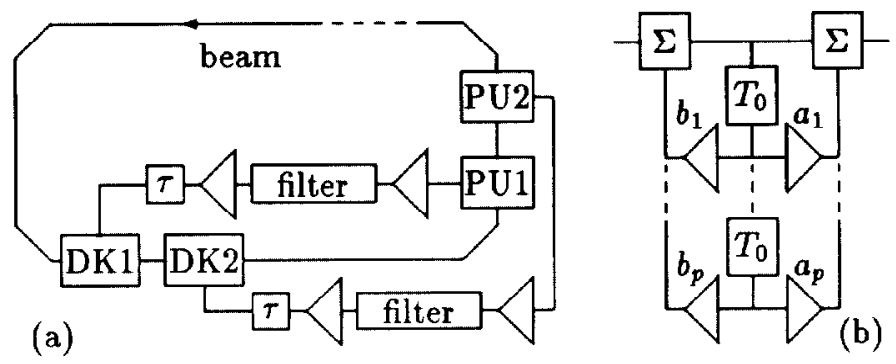

Figure 1: Feedback layout (a) and IIR-filter scheme (b).

becomes suitable for the beam motion description. Let the column matrix $\widehat{X}[n, s]$ determine the beam state at the $n$-th turn at point $s$ of the circumference $C_{0}$. The first element of this matrix equals the beam deviation $x[n, s]$ from the closed orbit and the second one is $x^{\prime}[n, s]$. After a short DK the $x^{\prime}$ value of the beam is changed by $\Delta x^{\prime}\left[n, s_{K}\right]$ while deviation remains the same as before the DK at point $s_{K}^{-}$. Hence, after DK at point $s_{K}^{+}$, the beam state is

$$
\widehat{X}\left[n, s_{K}^{+}\right]=\widehat{X}\left[n, s_{K}^{-}\right]+\widehat{T} \Delta \widehat{X}\left[n, s_{K}\right],
$$

where $\widehat{T}$ is the $2 \times 2$ matrix in which $T_{21}=1$ and the other elements are zero. The kick is determined with column matrix $\Delta \widehat{X}\left[n, s_{K}\right]$, where the first element equals $\Delta x^{\prime}\left[n, s_{K}\right]$ and the second one has an arbitrary value.

If $\widehat{M}\left(s_{2}, s_{1}\right)$ is the transfer matrix from $s_{1}$ to $s_{2}$, then at the PU1 location at the $(n+1)$-th turn the beam state is

$$
\begin{aligned}
& \widehat{X}\left[n+1, s_{P 1}\right]=\widehat{M_{0}} \widehat{X}\left[n, s_{P 1}\right]+ \\
&+\sum_{l=1}^{M} \widehat{M}\left(s_{P 1}+C_{0}, s_{K l}\right) \widehat{T} \Delta \widehat{X}\left[n, s_{K l}\right]
\end{aligned}
$$

where $\widehat{M}_{0}$ is the unperturbed revolution matrix from point $s_{P 1}$ of the PU1 location and $M$ is a number of kickers.

Let $\Delta x^{\prime}\left[n, s_{K l}\right]$ be proportional to the output voltage $V_{\text {out }}\left[n, s_{K I}\right]$ in the feedback circuit during $n$-th crossing of the $l$-th kicker. The input voltage $V_{i n}\left[n, s_{P l}\right]$ is assumed to be proportional to the beam deviation $x\left[n, s_{P I}\right]$ in the l-th pick-up. The kicker should change the angle of the same fraction of the beam that was measured by the PU. The delay $\tau=q T_{0}+\tau_{l}$ is adjusted to provide such a synchronization ( $q$ is integer, $T_{0}$ is the revolution period, $\pi$ is 
the time of the particle flight between PUl and DKl). If the kick at the $n$-th turn depends on the beam state in the previous turns, then for the digital circuit we can write [7]:

$$
V_{\text {out }}\left[n, s_{K^{\prime} l}\right]=u[n-q] \sum_{m=0}^{n-q} h[m] V_{\text {in }}\left[n-m-q, s_{P l}\right]
$$

where $u[n]$ is the discrete unit step function [7] and the $h[m]$ coefficients are determined in accordance with the structure scheme of the feedback circuit. If $s_{K 1}>s_{P M}$ and all feedback circuits are identical, then we have

$$
\begin{aligned}
& \Delta \widehat{X}\left[n, s_{K l}\right]=u[n-q] \times \\
& \quad \times \sum_{m=0}^{n-q} \frac{|\mathbf{K}| h[m]}{\sqrt{\beta_{P l} \beta_{K l}}} \widehat{M}\left(s_{P l}, s_{P 1}\right) \widehat{X}\left[n-m-q, s_{P 1}\right],
\end{aligned}
$$

where $\beta_{P l}$ and $\beta_{K l}$ are the transverse betatron amplitude functions in the PU and $D K$ locations and $|K|$ is the gain of the feedback without a filter.

Eqs. $(1,2)$ fully describe the beam dynamics in the accelerator with the digital feedback system. These equations can be solved using $Z$-transform [7] for sequence $\widehat{X}[n, s]$ :

$$
\begin{aligned}
\hat{\mathbf{X}}(z) & =\sum_{n=0}^{\infty} \hat{X}[n, s] z^{-n} ; \\
\hat{X}[n, s] & =\frac{1}{2 \pi i} \int_{C} \hat{\mathbf{X}}(z) z^{n-1} d z=\sum_{k} \operatorname{Rez}\left[\widehat{\mathbf{X}}\left(z_{k}\right) z_{k}^{n-1}\right] .
\end{aligned}
$$

The motion of the particles will be stable if $\left|z_{k}\right|<1$. The damping factor $D_{k}=\left|z_{k}\right|$ and the number of oscillations per turn $\left\{\operatorname{Re} Q_{k}\right\}=\arg \left(z_{k}\right) / 2 \pi$ are fully determined by the singular points $z_{k}$. Using $Z$-transform for $(1,2)$ we get:

$$
\begin{aligned}
\widehat{\mathbf{X}}(z) & =\frac{z \widehat{I}-\widehat{\mathbf{M}}^{-1}(z) \operatorname{det} \widehat{\mathbf{M}}(z)}{\operatorname{det}(z \widehat{I}-\widehat{\mathbf{M}}(z))} z \widehat{X}\left[0, s_{P 1}\right] \\
\widehat{\mathbf{M}}(z) & =\widehat{M}_{0}+ \\
& +\sum_{l=1}^{M} \frac{\mathbf{K}(z)}{\sqrt{\hat{\beta}_{P l} \beta_{K l}}} \widehat{M}\left(s_{P 1}+C_{0}, s_{K l}\right) \widehat{T} \widehat{M}\left(s_{P l}, s_{P 1}\right) \\
\mathbf{K}(z) & =z^{-q}|\mathbf{K}| H(z),
\end{aligned}
$$

where $\hat{I}$ is the unil matrix; $\widehat{X}\left[0, s_{P_{1}}\right]$ is the initial beam state matrix; $\mathbf{K}(z)$ is the transfer function for a feedback circuit with the delay $\tau$ and the filter system function $H(z)$ depending on $h[m]$. It is known [7] that in radiotechnical sense the circuit is stable if all the singular points of $\mathbf{K}(z)$ lie inside the circle $|z|<1$. If this condition is fulfilled, the singular points $z_{k}$ in (4) are found from the equation [8]:

$$
\begin{aligned}
& \operatorname{det}(z \widehat{I}-\widehat{\mathbf{M}}(z))=z^{2}-[2 \cos (2 \pi Q)+ \\
& \left.+\mathbf{K}(z) \sum_{l=1}^{M} \sin \left(2 \pi Q-\psi_{P I K l}\right)\right] z+\operatorname{det} \widehat{\mathbf{M}}(z)=0,
\end{aligned}
$$

where $Q$ is the number of unperturbed betatron oscillations per revolution in transverse plane; $\psi_{P I K l}$ is the betatron phase advance from PUl to DKl.
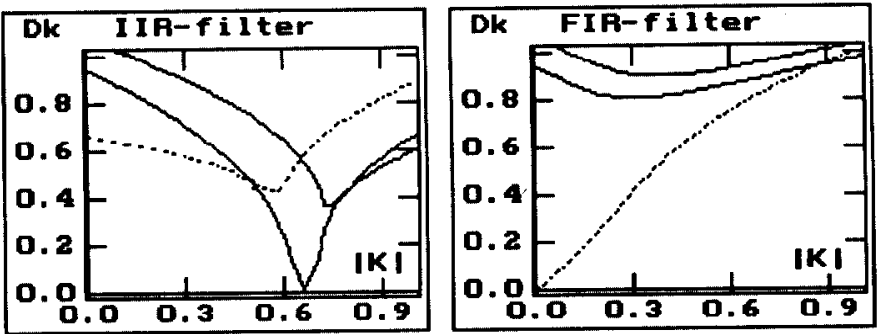

Figure 2: $D_{k}$ for a classical feedback with IIR-filter (left) and FIR-filter (right). $\quad \operatorname{Re} Q=70.3 ; \quad|\operatorname{Im} Q|=0.01$.

When instability occurs, Eq.(6) will have the same form but the betatron phase advances must be calculated with a complex value of $Q(z)$ both for coasting [6] and bunched [9] beams.

\section{Feedback With Filter}

The digital IIR-filter (Fig.1b) consists of amplifiers $a_{m}$, $b_{m}$ and delays $T_{0}$; the order of the filter is $p$. The system function for this filter is

$$
H(z)=\left[1+\sum_{m=1}^{p} a_{m} z^{-m}\right]\left[1-\sum_{m=1}^{p} b_{m} z^{-m}\right]^{-1} .
$$

The results for the filter of the first order $(p=1)$ and for $q=0$ are shown bellow.

\section{A. Classical Feedback}

For a classical feedback we have $M=1$ and

$$
\operatorname{det} \widehat{\mathbf{M}}(z)=1-\mathbf{K}(z) \sin \psi_{P K} .
$$

Taking into account $(5,7)$ we get in $(6)$ a cube equation for $z_{k}$. If $|\mathbf{K}| \ll 1$, then in linear approximation we obtain:

$$
\begin{aligned}
z_{1,2} & =\left(1 \mp \frac{i}{2}|\mathbf{K}| e^{\mp i \psi_{P K}}\right) e^{ \pm i 2 \pi Q}-\frac{a_{1}+b_{1}}{2}|\mathbf{K}| \mu_{1,2} \\
z_{3} & =b_{1}+\left(a_{1}+b_{1}\right)|\mathbf{K}| \mu_{3}
\end{aligned}
$$

where $\mu_{i}$ are defined in [6]. Without a filter $\left(a_{1}=b_{1}=0\right)$ we have two solutions and a damping time $\tau_{D}$ is

$$
\begin{aligned}
\frac{T_{0}}{\tau_{D}} & =-\ln \mathrm{MAX}\left|z_{k}\right|=\frac{1}{2}|\mathrm{~K}| \sin \left(\operatorname{Re} \psi_{P K}\right)-2 \pi|\operatorname{Im} Q|= \\
& =\frac{1}{2} \sqrt{\beta_{P} \beta_{K}}\left(\frac{\Delta x_{K}^{\prime}[n]}{x_{P}[n]}\right) \sin \left(\operatorname{Re} \psi_{P K}\right)-2 \pi|\operatorname{Im} Q| .
\end{aligned}
$$

This decrement formula is well known [4]. However, for a feedback with a filter it is necessary to take into account three roots. Fig.2 shows $\left|z_{k}\right|$ dependencies on $|\mathbf{K}|$ when the phase advance from PU to DK is adjusted closely to an odd number of $\pi / 2$ radians $\left(\left|\sin \operatorname{Re} \psi_{P K}\right|=1\right)$. The solid curves correspond to the oscillations with the tune in neighbourhood of $Q$. The dotted curve corresponds to 

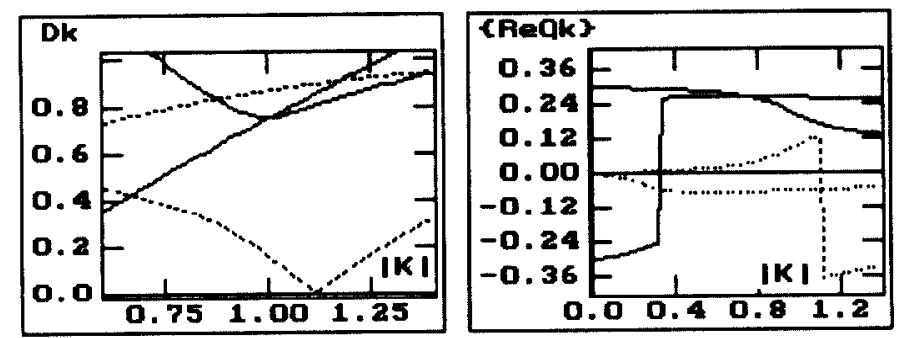

Figure 3: $D_{k}$ and $\left\{\operatorname{Re} Q_{k}\right\}$ for a fast feedback.

the third root. This new oscillation mode is conditioned with the filtcr structure, when the kick in DK depends on the beam state in the previous two revolutions. To provide the independence on $|\mathbf{K}|$ of the feedback action on the closed orbit displacement and for a better suppression of noise it is necessary to set $a_{1}=-1$ [6]. Optimization of $b_{1}$ value on the maximum damping rate gives $b_{1}=0.66$ for a feedback with the IIR-filter. It is seen from Fig.2 (left) that for $|\mathbf{K}|>\mathbf{0 . 6 5}$ the damping rate is determined by third oscillation mode. For $b_{1}=0$ we have a notch filter (FIR-filter). The maximum decrement in this case (Fig.2, right) corresponds to $|\mathbf{K}|=0.38$. It is easy to see that a feedback system with a notch filter is slower and its stability region is narrower than the feedback system with the IIR-filter.

\section{B. Fast Feedback}

For a fast feedback we have $M=2$. If $\operatorname{Re} \psi_{P 2 P 1}=$ $\operatorname{Re} \psi_{K 2 K 1}=\pi / 2$, then $^{1}$

$$
\operatorname{det} \widehat{\mathbf{M}}(z)=1-2 \mathbf{K}(z) \sin \psi_{P K}+\mathbf{K}^{2}(z) .
$$

After some transformations in (6) we get the equation:

$$
z-\left[1 \mp i \mathbf{K}(z) \exp \left(\mp i \psi_{P K}\right)\right] \exp ( \pm i 2 \pi Q)=0 .
$$

Hence, for $\mathbf{K}(z)$ in (5) with $H(z)$ from (7) the additional solutions $z_{k}$ and, thus, the new eigen frequencies are obtained. This leads to modification of stability region, $\left|z_{k}\right| \leq 1$, especially when $|\mathbf{K}| \geq 1$. The maximum damping rate is achieved by the fast feedback system at optimum positions of $\mathrm{PU}$ and $\mathrm{DK}\left(\left|\sin \operatorname{Re} \psi_{P K}\right|=1\right)$ connected via feedback without filter [3] and its value is

$$
\operatorname{MAX}\left(D_{k}\right)=|\sinh (2 \pi \operatorname{Im} Q)| \quad \text { for }|\mathbf{K}|=\cosh (2 \pi \operatorname{Im} Q) .
$$

This means that without instability the fast TFS can damp the coherent oscillations in one turn. But in the UNK-1 it is expected that $|\operatorname{Im} Q|=0.1$. Hence, the stability region is not large. For this reason the $\mathrm{PU}$ and DK positions were taken as close to their optimum positions as possible. In Fig. 3 the $D_{k}$ and $\left\{\operatorname{Re} Q_{k}\right\}$ dependencies on $|\mathbf{K}|$ are shown for the feedback system with the IIR-filter. As it is mentioned above there are more than two ordinary eigen

\footnotetext{
'The influence of deviations for phase advances from $\pi / 2$ radians is analyzed in [8].
}

frequencies for the feedback with a filter. In Fig. 3 the solid curves correspond to the ordinary modes and the dotted curves are determined by the filter structure. For the same reasons mentioned above for a classical feedback it is necessary to set $a_{1}=-1$. The filter parameter $b_{1}=0.61$ was chosen to provide the best damping conditions. The $\operatorname{Re} Q$ value for all these curves is 56.7 . But with a different number of particles the coherent tune shift for $\operatorname{Re} Q$ may be about 0.09 for the horizontal and 0.41 for vertical betatron oscillations [2]. It means that $\operatorname{Re} \psi_{P K}$ will differ from its optimum value. The particle motion is stable, if [3]

$$
\left|\sin \left(\operatorname{Re} \psi_{P K}\right)\right|>|\sinh (2 \pi \operatorname{Im} Q)| .
$$

Hence, the Re $\psi_{P K}$ deviation is not more than $0.2 \pi$ radians in the UNK-1. As soon as this deviation is less than the coherent tune shift for vertical oscillations, then the tune must be corrected during injection in order to have an acceptable phase advance from PU2 to DK1.

It is necessary to emphasize that the optimum value of $b_{1}$ depends on $Q$. For this reason in the filter design it is useful to foresee a possibility to vary the $b_{1}$.

\section{Conclusion}

The matrix equations for a transverse feedback system with a digital filter have been obtained and the $Z$-transform method has been effectively used to solve them. The analytical solutions for the damping time and for the eigen frequencies obtained allow one to design feedback systems with digital filters and to optimize the damping rate and the amplifier gain in stability region.

\section{ACKNOWLEDGMENTS}

The authors would like to thank I.N. Ivanov (JINR) and D. Boussard (CERN) for help and many useful discussions.

\section{REFERENCES}

[1] L. Vos. CERN SL/91-40 (BI), Geneva, 1991.

[2] V.I. Balbekov. The IX All Union Conference on Particle Accelerators. JINR, Dubna, vol.2, p.360, 1985.

[3] V.M. Zhabitsky et al. JINR P9-91-99, Dubna, 1991.

[4] E. Ebert et al. DESY 91-036, 1991.

[5] V.M. Zhabitsky et al. JINR P9-91-494, Dubna, 1991.

[6] V.M. Zhabitsky. CERN SPS/RFS/91-14, Geneva, 1991.

[7] W.McC. Siebert. Circuits, Signals, and Systems. The MIT Press, 1986.

[8] V.M. Zhabitsky. JINR P9-92-310, Dubna, 1992.

[9] V.M. Zhabitsky et al. JINR P9-92-309, Dubna, 1992. 